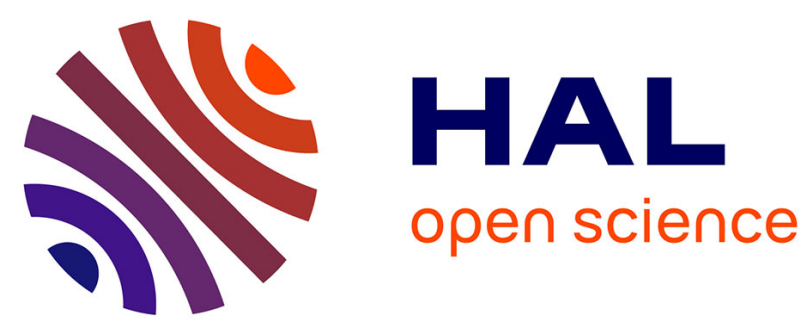

\title{
Étude comparée de quatre indicateurs qualifiant le champ visuel d'un piéton en milieu urbain
}

Thomas Leduc, Guillaume Chauvat

\section{To cite this version:}

Thomas Leduc, Guillaume Chauvat. Étude comparée de quatre indicateurs qualifiant le champ visuel d'un piéton en milieu urbain: Partitionnement de l'espace ouvert urbain et représentation de la prévisibilité d'un environnement urbain. Revue Internationale de Géomatique, 2015, Traitement de l'information et prospective, 25 (1), pp.75-98. 10.3166/rig.25.75-98 . hal-01150201

\section{HAL Id: hal-01150201 \\ https://hal.science/hal-01150201}

Submitted on 9 May 2015

HAL is a multi-disciplinary open access archive for the deposit and dissemination of scientific research documents, whether they are published or not. The documents may come from teaching and research institutions in France or abroad, or from public or private research centers.
L'archive ouverte pluridisciplinaire HAL, est destinée au dépôt et à la diffusion de documents scientifiques de niveau recherche, publiés ou non, émanant des établissements d'enseignement et de recherche français ou étrangers, des laboratoires publics ou privés. 


\title{
Étude comparée de quatre indicateurs qualifiant le champ visuel d'un piéton en milieu urbain
}

\author{
Partitionnement de l'espace ouvert urbain et \\ représentation de la prévisibilité d'un environnement \\ urbain
}

\author{
Thomas Leduc ${ }^{1}$, Guillaume Chauvat ${ }^{2}$ \\ 1. Laboratoire CERMA, UMR CNRS 1563, École Nationale Supérieure \\ d'Architecture de Nantes \\ 6 quai François Mitterrand, BP 16202, F-44262 Nantes cedex 2, France \\ thomas.leduc@cerma.archi.fr \\ 2. École Nationale Supérieure d'Architecture de Nantes \\ 6 quai François Mitterrand, BP 16202, F-44262 Nantes cedex 2, France \\ chauvat.guillaume@gmail.com
}

\begin{abstract}
RESUME. Pour la théorie de la perméabilité, la configuration des lieux, et plus précisément la présence d'occlusions dans notre environnement visuel, peuvent nous inciter à son exploration. L'étude présentée dans cet article a pour but d'analyser de manière systématique et reproductible la prévisibilité de l'environnement urbain immédiat d'un piéton. Pour évaluer cette propriété du champ visuel en tout point, nous rappelons trois indicateurs permettant de quantifier la prévisibilité de la forme ambiante (entropie), le poids des masses visuelles (excentricité) et la part d'occlusions (occlusivité) avant d'en proposer un quatrième relativisant le poids de chaque occlusion (l'anticipation). L'agrégation opérée par un partitionnement convexe de l'espace ouvert facilite l'émergence de motifs géométriques caractérisables pour une meilleure compréhension des potentialités de l'espace urbain.

ABSTRACT. According to the permeability theory, the spatial configuration, and more precisely, the presence of occlusions in our surroundings may invite us to explore our environment. This article aims to analyze, in a systematic and reproducible way, the predictability of our visual environment. To assess this property for every observing point, we remind three indicators relative to the boundary predictability (entropy), to the weight of the visible mass (drift), and to the occlusions ratio (occlusivity). We introduce a fourth indicator weighting each visual discontinuity (anticipation). An aggregation operated thanks to the open space convex partition emphasizes characterisable patterns, towards an application for urban planning.
\end{abstract}


MoTS-CLES : isovist, entropie, occlusivité, excentricité, anticipation, partitionnement convexe, $s$-segments, e-segments, exploration environnementale, morphologie

KEYWORDS: isovist, entropy, occlusivity, drift, anticipation, convex partition, s-lines, e-lines, environmental exploration, morphology

\section{Introduction}

Comme le rappelle (Brayer, 2013), la façon dont nous construisons les villes peut avoir un effet profond sur la manière dont nous les vivons. De ce constat a émergé un ensemble de préoccupations contemporaines portées aujourd'hui par la notion d'ambiance. Cette notion prend en compte les dimensions culturelles et conjoncturelles d'un lieu pour saisir son identité, parce que les pratiques d'un lieu ne sont pas réductibles à ses seules caractéristiques construites. Dans le même registre, pour (Péneau, 2000, p. 379), la seule approche des dimensions physiques ne suffit pas à rendre compte de la consistance phénoménologique des ambiances. Le sujetcitadin est ainsi marqué par toute une gamme de différenciateurs culturels, psychologiques et sociaux, représentant autant de filtres à la perception des phénomènes objectifs tels que la température ambiante, les rayonnements, les vitesses d'air, etc. Pour le concepteur d'environnements urbains, ce constat est lourd d'implications. Pour le bureau d'étude spécialisé dans certaines des dimensions phénoménales impliquées dans le processus de projet ou le modélisateur, il est implacable. La modélisation et la simulation d'une ou plusieurs dimensions physiques d'un environnement urbain ne suffisent pas à expliquer, seules, la complexité intrinsèque du genius loci.

Cette précaution ayant été prise, nous pouvons néanmoins reconnaître avec (Stamps III, 2010) que la configuration géométrique et topologique des lieux peut encourager ou inciter le piéton à l'exploration de son environnement immédiat. Pour (Hall, 1966), la présence de discontinuités dans cet environnement visible influe sur nos intentions de déplacement. Pour (Kaplan, 1973), évaluer la part de mystère d'une scène signifie être en mesure de répondre à la question suivante : To what degree do you think you would learn more if you could walk deeper into the scene? Plus précisément, pour (Cullen, 1961), il y a une promesse de gain d'information si le sujet a la possibilité de rejoindre un meilleur point de vue, c'est-à-dire encore si l'objet occlusif qui s'intercale entre le point de vue et l'arrière plan caché peut-être rejoint et offrir ainsi une nouvelle perspective sur la part d'ombre du paysage (ce qu'il appelle the sense of mystery). En fait, plus même que de mystère, la bonne terminologie, pour (Cullen, 1961), semble être l'anticipation. Il met ainsi en perspective le proche (l'« ici ») et le lointain (le «là-bas ») où l'« ici » est connu et le « là-bas» ne l'est pas et énonce que s'engager sur un nouveau tronçon est plus motivant si nous ne savons pas à l'avance ce qui nous attend (The next stretch is more inviting if we don't know completely what awaits us).

La part cachée d'un paysage n'est cependant pas que stimulante ou attirante. Elle peut aussi être génératrice d'anxiété. (Stamps III, 2005) développe ainsi une théorie de la perméabilité selon laquelle le contour de l'espace ouvert immédiatement 
environnant est étroitement corrélé à l'impression de sécurité du sujet-piéton. Il distingue alors les frontières matérielles ou solides (en l'occurrence les façades opaques qui arrêtent le regard et préviennent toute pénétration dans l'environnement visuel immédiat), des frontières occlusives (immatérielles) qui résultent d'un effet de masque et cachent une part d'espace imprévisible.

La compréhension de l'environnement urbain ambiant par le sujet-piéton n'est pas seulement fonction de la physique des lieux ou d'une dimension psychologique. Une reconfiguration mentale de la structure spatiale des lieux, que l'on nomme une représentation cognitive de l'espace, résulte de l'interaction entre le sujet, l'environnement qu'il traverse ou habite, et l'action ou la séquence d'actions qui lie le sujet à son milieu (Cauvin, 1999). La dimension cognitive est essentielle dans l'élaboration de stratégies d'orientation ou de déplacement du piéton. Elle est aussi prépondérante dans l'évaluation des distances.

La prévisibilité d'un environnement est extraite de l'information qui y est perceptible, qu'elle soit liée à une frontière solide ou occlusive. L'étude que nous restituons ici a pour but d'analyser de manière systématique, objective et reproductible la prévisibilité d'un paysage urbain. Les enjeux de cette recherche sont triples. Tout d'abord, l'identification des potentialités associées aux spécificités locales de forme génère un diagnostic. Ces données, corrélées avec une partition de l'espace visible, offrent par la suite des éléments de compréhension de l'environnement étudié, finalement mis au service d'un aménagement cohérent du lieu.

Cette analyse, pour pouvoir être transposée à d'autres terrains, met en œuvre un modèle qui exploite des jeux de donnée vectorielle standards, dans le contexte d'un Système d'Information Géographique (SIG). L'outil méthodologique que nous avons retenu est le champ visuel ou visualscape défini dans (Llobera, 2003, p. 30) comme la représentation spatiale de toute propriété visuelle générée par, ou associée à, une configuration spatiale des lieux. On attribue traditionnellement les premières formalisations et représentations de champ visuel à (Benedikt, 1979) qui a introduit la notion d'isovist (polyèdre - ou polygone si l'on travaille dans un plan 2D - formé de l'ensemble des portions de l'espace visibles depuis un lieu d'observation donné) et de champ d'isovists. Un champ d'isovists est, pour Benedikt, un champ scalaire, qui associe à tout point de l'espace (discrétisé) une grandeur qui représente l'environnement visible immédiat. Comme le remarque (Morello et Ratti, 2009), le champ d'isovists offre une perspective synthétique, sous forme cartographique, de l'ensemble des valeurs d'une même propriété visuelle en tout point du terrain d'étude.

Pour analyser la prévisibilité du champ visuel en tout point de l'espace d'étude, nous utilisons quatre indicateurs. Trois d'entre eux sont issus de la bibliographie du domaine. Ils permettent de quantifier respectivement la prévisibilité de la forme (notion empruntée à la théorie de l'information), le «poids des masses visuelles » ou la part d'occlusions dans le bassin de visibilité. Le quatrième, développé au cours d'une étude réalisée au sein du laboratoire CERMA, s'attache à mesurer l'importance visuelle de ce qui échappe à notre champ de vision, par un contraste de plans. Ce dernier a plus particulièrement été formulé en vue d'une analyse des motivations 
intrinsèques au déplacement du piéton en situation de «mouvement naturel » (Hillier et al., 1993).

Afin de nous affranchir de l'arbitraire de l'échantillonnage ponctuel de l'espace ouvert, de donner une réalité spatiale aux quatre indicateurs introduits dans le paragraphe précédent et de faciliter une lecture synthétique des résultats, nous avons cherché une solution d'agrégation des points de mesure. Pour cet opérateur d'agrégation, notre choix s'est porté sur le partitionnement convexe de l'espace visible. En effet, selon (Peponis et al., 1997) qui a présenté les notions de s-partition et e-partition, ces tessellations de l'espace ouvert urbain permettent de le diviser en sous-espaces d'information visuelle stable.

Dans cet article, après une présentation détaillée et motivée de la notion de champ d'isovists et du mode de calcul retenu pour l'évaluer, nous introduirons les quatre indicateurs que nous avons choisis pour analyser la part d'inconnu d'un environnement urbain. Cette définition sera complétée par l'énoncé des enjeux et des principes du partitionnement convexe de l'espace visible. La méthodologie ayant été exposée, nous l'appliquerons à un terrain d'étude dans le centre de Nantes (France), puis discuterons ses apports notamment au regard d'une possible application à la conception de l'environnement urbain.

\section{Méthode}

\subsection{Construction du champ visuel}

L'analyse systématique de champ visuel urbain que nous voulons conduire requiert un outil logiciel robuste et performant. Le calcul de champs scalaires relatifs à un bassin de visibilité nécessitant un développement dédié, nous utilisons, pour le traitement spatial, l'instance libre de SIG Gearscape ${ }^{1}$. Ce développement exploite des bibliothèques de traitement spatial standard $\left(\mathrm{JTS}^{2}\right.$ et GeoTools $\left.{ }^{3}\right)$ associées à une bibliothèque propre à cette instance de SIG. Un découplage de cette dernière en faciliterait la diffusion. S'agissant de la visualisation, nous avons utilisé le logiciel libre QGIS. Concernant l'environnement urbain ou plus exactement sa maquette, nous exploitons la couche bâtiments de la BD TOPO®.

\subsubsection{Hypothèses}

Nous faisons l'hypothèse que toutes les façades sont opaques et n'intégrons, dans notre modèle, ni les transparences, ni les divers obstacles au champ de vision comme la végétation, le mobilier urbain, etc. Le champ de vision que nous modélisons est périphérique $2 \mathrm{D}$, il n'est en aucun cas directionnel dans la mesure où

${ }^{1}$ GearScape est disponible en ligne à l'adresse http://www.gearscape.org/.

2 La JTS Topology Suite est une interface de programmation disponible en ligne à l'adresse http://sourceforge.net/projects/jts-topo-suite/.

${ }^{3}$ GeoTools The Open Source Java GIS Toolkit est une interface de programmation disponible en ligne à l'adresse http://www.geotools.org/. 
nous cherchons à qualifier un ressenti d'espace ouvert immédiatement environnant plus qu'un champ de vision réaliste. Ne disposant pas de données altimétriques suffisamment précises et afin de limiter la complexité algorithmique, nous n'avons pas modélisé de bassin de visibilité 3D. Il est néanmoins envisageable de le formuler en $3 \mathrm{D}$ sous réserve de disponibilité de données parfois imprécises (les hauteurs à l'épannelage) ou difficiles à obtenir et à mettre à jour (les types de toits, pentes des toitures, etc.). Ce travail complémentaire permettrait de prendre en compte de manière plus réaliste la topographie du cas d'étude, d'appréhender l'influence de la hauteur du bâti, de l'inclinaison du terrain. La densité du bâti diminuant, la topographie aurait un impact d'autant moins négligeable. Les indicateurs présentés ici pourraient alors être éprouvés en milieu urbain plus diffus voire même hors du milieu urbain.

\subsubsection{Principe de construction}

Pour (Joliveau, 2004, p. 121), des fonctions d'analyse de visibilité ont été implémentées dans les SIG sous le nom de viewshed analysis. Elles utilisent couramment des Modèles Numériques de Terrain (MNT) ou des réseaux de triangles irréguliers (TIN) et évaluent l'espace visible depuis des cibles matérialisées sous forme de points vecteurs ou de cellules de grille raster. Par ailleurs, plusieurs approches pour délimiter automatiquement l'espace visible d'un piéton en milieu urbain reposent sur des données vectorielles (Suleiman et al., 2012). La plus connue, le lancer de rayon, consiste à évaluer l'intersection entre un ensemble de rayons équi-répartis autour du point de vue (3600 pour l'implémentation de (Rana, 2006) dans ArcView) et les premiers obstacles de l'environnement proche. Une variante de cette méthode, dite de l'agent en marche (Batty et Jiang, 1999), consiste non seulement à discrétiser l'azimut comme dans la méthode du lancer de rayon, mais aussi à discrétiser la distance au point de vue. (Turner et al., 2001) ont proposé une troisième méthode consistant à discrétiser préalablement l'espace d'étude et à procéder en tout point à des évaluations d'intervisibilités avec l'ensemble des autres points du maillage.

\subsubsection{Calcul « exact» de l'isovist en $2 D$}

Comme le constate (Suleiman et al., 2012), l'évaluation du bassin de visibilité en milieu urbain par l'une des approches mentionnées ci-avant dépend du nombre de rayons lancés, de la précision du « pas de l'agent », du nombre de points de maillage, ou de la résolution du pixel. L'environnement urbain étant susceptible (dans les tissus irréguliers du moins) de présenter une grande complexité du déroulé de façades, nous avons opté pour une approche «exacte». Par «exacte», nous entendons ici que la méthode retenue ne procède pas par discrétisation de l'espace, par discrétisation par abscisse angulaire, ou par discrétisation des arêtes opaques. Nous travaillons dans un espace vectoriel continu (aux limitations de l'arithmétique flottante IEEE 754 de la machine virtuelle Java près). Calquée sur le constat de (Suleiman et al., 2012), notre implémentation fait l'hypothèse que tout fragment urbain peut être assimilé à un ensemble de segments sans intersection sinon 
éventuellement aux extrémités. Elle reprend et adapte ensuite l'algorithme de détermination des arêtes cachées d'une chaîne graphique classique :

- élimination des faces ou arêtes cachées à partir de l'orientation des normales aux faces (backface culling),

- élimination des faces ou arêtes hors du champ ou cône de vision (viewing frustum culling). Comme la vision est périphérique sur $360^{\circ}$, nous ne pouvons en l'occurrence pas parler de cône de vision. Sont donc éliminés lors de cette phase tous les objets trop distants (pour des raisons d'efficacité, nous exploitons ici les index spatiaux de JTS). Au cours de cette même phase, nous procédons à un clipping. Celui-ci consiste à découper les arêtes partiellement visibles ;

- élimination des faces ou arêtes qui se cachent mutuellement (occlusion culling). D'un point de vue pratique, nous recherchons les points minimum (minimum de potentiel) dans une représentation polaire (centrée sur le point de vue) des arêtes.

La quatrième et dernière phase d'élimination des objets minuscules (contribution culling) n'est volontairement pas implémentée dans notre processus.

\subsection{Formulation de quatre indicateurs qualifiant le champ visuel}

2.2.1. L'entropie de Shannon de la fonction de distances radiales comme indicateur de prévisibilité du bassin de visibilité

L'isovist, associé à un point de vue, est un polygone qui présente la particularité de contenir tout segment reliant chacun de ses points à ce même point de vue (appelé aussi point de génération). Cette propriété topologique permet de simplifier l'étude de l'isovist, en la réduisant à l'analyse de son contour. Nous pouvons donc caractériser ce polygone par l'étude de la fonction d'une variable réelle $2 \pi$ périodique qui, à un azimut donné (l'abscisse angulaire), associe la longueur du rayon reliant le point de vue au point de contour correspondant (cf. figure 1).

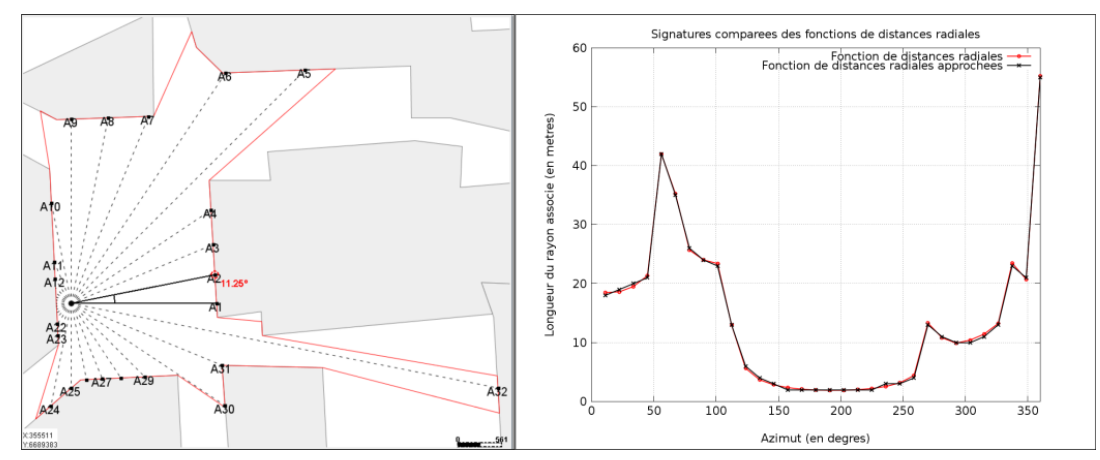

Figure 1. (gauche) Représentation en plan, pour un fragment urbain (les empreintes polygonales de bâtiments sont en gris clair), d'un point de vue et de son isovist. (droite) Développé en fonction de distances radiales correspondant. 
Cette fonction mathématique d'une variable réelle peut alors être étudiée à l'aide d'un outil de la théorie de l'information. Comme rappelé par (Salat, 2011, p. 491), la formule de l'entropie de Shannon (cf. équation 1), en quantifiant la quantité d'information contenue dans une distribution, est une façon de quantifier la complexité de cette dernière.

$$
H=-\sum_{i=1}^{n} P_{i} \times \log _{b} P_{i}
$$

Dans cette équation, nous considérons que la longueur des rayons de la fonction de distances radiales est une variable aléatoire discrète (pour ce faire l'ensemble des longueurs est préalablement échantillonné en $\mathrm{b}$ intervalles). La grandeur $\mathrm{P}_{\mathrm{i}}$ correspond à la probabilité d'occurrence de la longueur du i-ème rayon, dans l'ensemble des valeurs de longueurs de rayons. L'entropie de la fonction de distances radiales de l'isovist d'un observateur placé au centre d'un espace ouvert parfaitement circulaire est nulle. En effet, dans cette configuration, tous les rayons visuels sont de même longueur, l'éloignement du point de vue au contour est parfaitement prévisible (car il est constant). En pratique, pour pouvoir calculer cette entropie, nous avons procédé à une discrétisation préalable des longueurs de rayons. Comme le montrent les signatures comparées de la figure 1, un échantillonnage avec une précision métrique ne modifie pas significativement la fonction des distances radiales.

\subsubsection{L'excentricité comme révélateur du "poids des masses visuelles »}

Le drift ou excentricité introduit par (Conroy Dalton et Dalton, 2001), correspond à la distance euclidienne séparant le point de vue du centre de masse de l'isovist correspondant (figure 2, équation 2). Cette grandeur permet de mesurer l'éloignement du centre visuel au centroïde de l'espace ouvert correspondant, c'est-àdire de quantifier l'impression éventuelle de l'observateur d'être ou non situé au centre de son bassin de visibilité.

$$
\text { excentricité }=\|\overrightarrow{O G}\|
$$

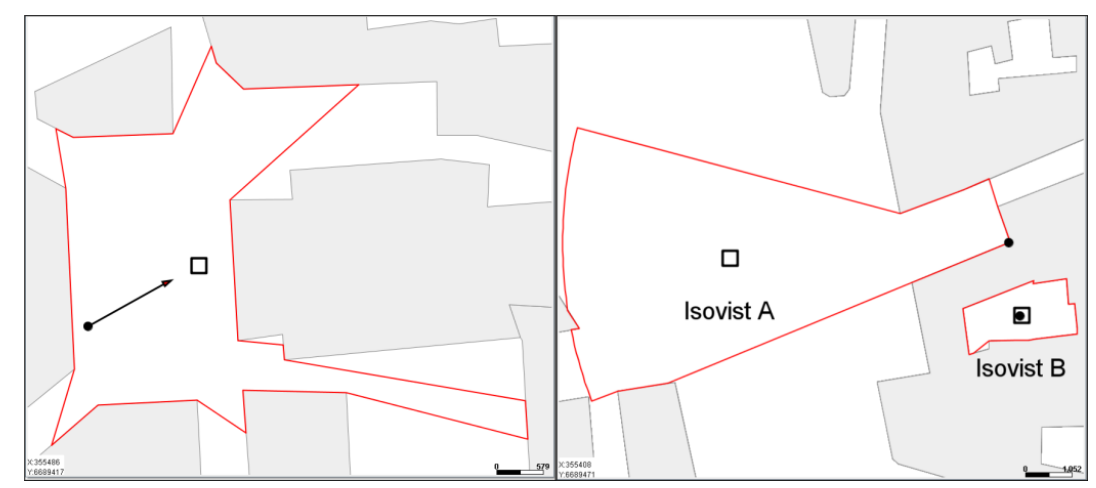

Figure 2. (gauche) L'excentricité du point d'observation (disque noir) est la distance qui le sépare du centre de gravité (carré évidé) de l'isovist correspondant. (droite) 
Deux situations extrêmes pour l'indicateur d'excentricité. L'excentricité de l'isovist A est de plus de $62 \mathrm{~m}$ (le point de vue, à son extrémité est, est très éloigné du centre de masse du bassin de visibilité). A contrario, l'excentricité de l'isovist B est inframétrique d'environ 0,45 m (point de vue et centre de masse sont presque confondus).

Pour (Leduc et Kontovourkis, 2012, p. 70), l'excentricité du point de vue dans son bassin visuel, est aussi une mesure de l'effort requis pour superposer ce point au centre de gravité de l'espace ouvert immédiatement associé.

\subsubsection{L'occlusivité comme ratio révélateur de la part d'inconnu du paysage}

L'occlusivité, (Benedikt, 1979), correspond au cumul des longueurs d'arêtes adjacentes à des portions d'espace ouvert invisibles depuis le point de vue (figure 3, équation 3). C'est un cumul de longueurs d'arêtes qui ne sont pas matérielles (et donc pas opaques). En pratique, la présence d'une occlusion se traduit par une concavité dans le bassin de visibilité correspondant. Cette notion est aussi régulièrement utilisée en aéraulique urbaine par des auteurs tels que (Adolphe, 2001; Edussuriya et al., 2011). Dans le registre de la visibilité, un tel indicateur permet de mesurer l'importance de ce qui échappe à notre champ de vision (donc de quantifier le poids des parties cachées d'un paysage).

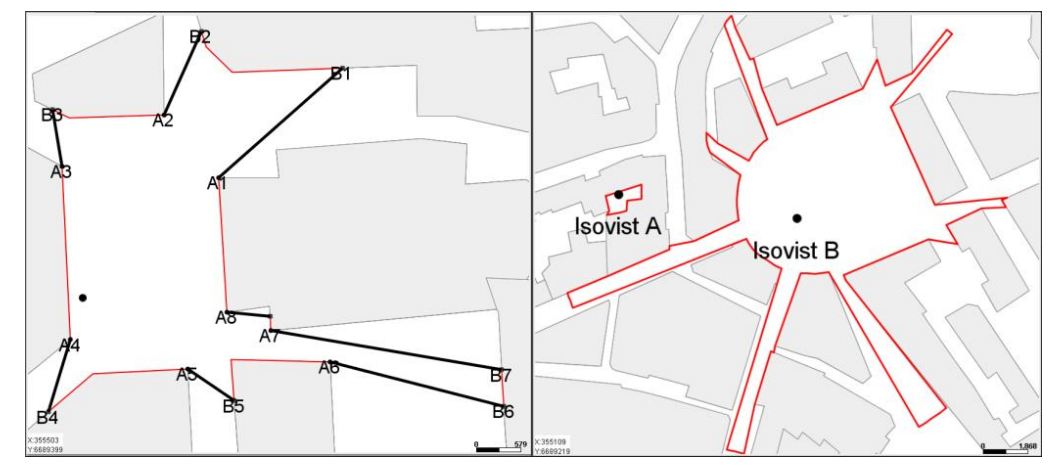

Figure 3. (gauche) L'occlusivité de O est la somme des longueurs des arêtes $A_{i} B_{i}$ qui sont occlusives car elles séparent le bassin de visibilité de zones d'espace ouvert invisibles depuis $O$. (droite) Deux situations extrêmes pour l'indicateur d'occlusivité. L'occlusivité de l'isovist A est approximativement de $2 \mathrm{~m}$, celle de l'isovist B est supérieure à $550 \mathrm{~m}$.

Si $\mathrm{A}_{\mathrm{i}} \mathrm{B}_{\mathrm{i}}$ est l'une des $\mathrm{k}$ arêtes occlusives de l'isovist dont on cherche à calculer la valeur de l'indicateur d'occlusivité, l'occlusivité est obtenue à l'aide de la formule suivante (la norme utilisée est la norme euclidienne) :

$$
\text { occlusivité }=\sum_{i=1}^{k}\left\|\overrightarrow{A_{l} B_{l}}\right\|
$$




\subsubsection{L'anticipation ou attraction des masses cachées du paysage}

Ce quatrième indicateur, appelé «anticipation » et développé lors d'une étude réalisée au sein du laboratoire CERMA (Chauvat, 2012), s'attache à évaluer l'influence relative des discontinuités présentes dans notre champ de vision. Contrairement à l'occlusivité, il pondère l'importance de chaque occlusion en fonction de sa distance au point de vue d'une part, de sa longueur d'autre part et de la distance du point de vue à l'arrière plan enfin. La formulation mathématique de cet indicateur est donnée en équation 4 (la norme utilisée est la norme euclidienne), comme on peut le constater, la distance du point de vue à l'occlusion est au dénominateur car l'impact visuel de l'occlusion décroit avec la distance. Une illustration de cet indicateur dans le cas d'une configuration urbaine nantaise réelle est donnée en figure 4.

$$
A=\sum_{i=0}^{n-1} \frac{\left\|\overrightarrow{O B_{l}}\right\| \times\left(\left\|\overrightarrow{O B_{l}}\right\|-\left\|\overrightarrow{O A_{l}}\right\|\right)}{\left\|\overrightarrow{O A_{l}}\right\|}=\sum_{i=0}^{n-1}\left(\frac{\left\|\overrightarrow{O B_{l}}\right\|^{2}}{\left\|\overrightarrow{O A_{l}}\right\|}-\left\|\overrightarrow{O B_{l}}\right\|\right)
$$

Cet indicateur est dimensionné (il s'exprime en mètre). Il n'est pas normalisé.

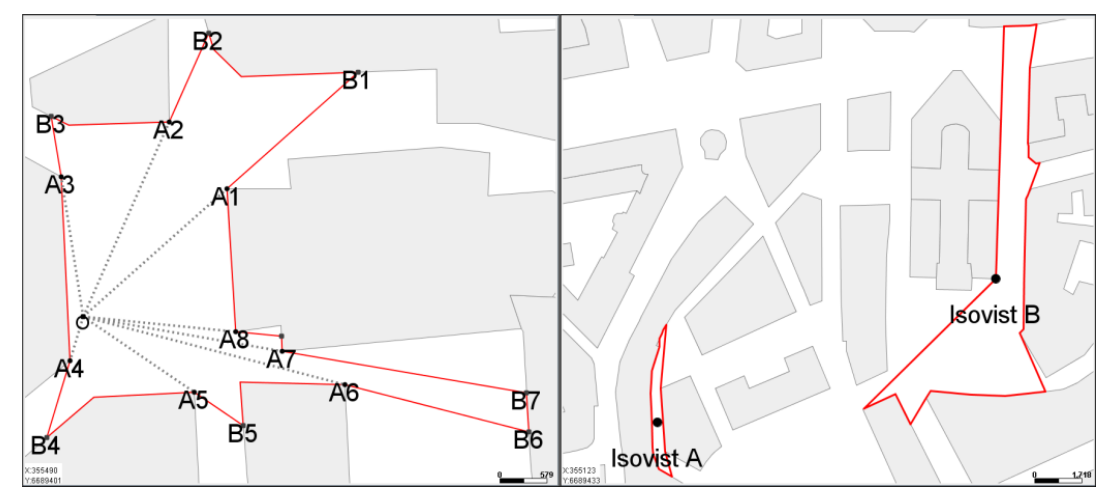

Figure 4. (gauche) Dans l'isovist associé au point de vue $O$, les points $A i$ matérialisent les huit occlusions associées à $O$ et les points Bi les arrières plans correspondants. (droite) Deux situations extrêmes pour l'indicateur d'anticipation.

L'anticipation de l'isovist A est de l'ordre du décamètre, celle de l'isovist B est supérieure à $268 \mathrm{~m}$.

La figure 5 présente, en chaque point d'espace ouvert de quelques configurations urbaines théoriques (bifurcation à angle droit, jonction en $\mathrm{T}$, jonction en $\mathrm{Y}$ ), des champs scalaires de valeurs d'anticipation. 

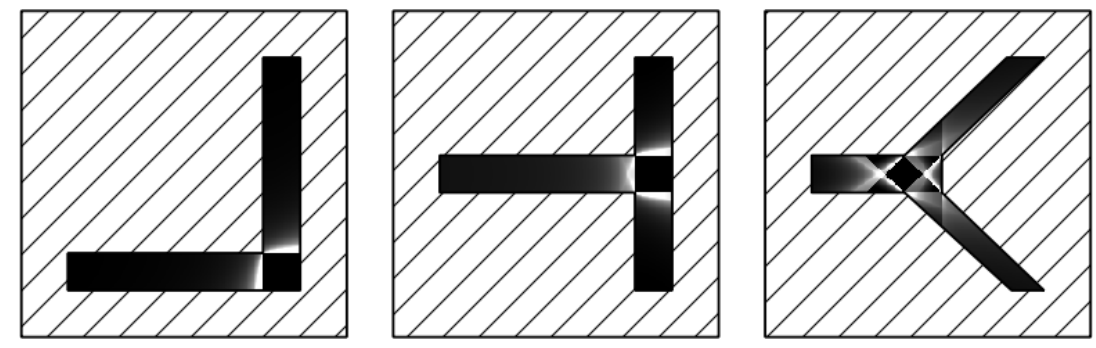

Figure 5. Champs scalaires de valeurs d'anticipation pour trois configurations urbaines théoriques (l'espace bâti est hachuré). La symbologie procède du plus sombre (faibles valeurs, anticipation minimale) au plus clair (fortes valeurs, anticipation maximale).

\subsection{Partitionnement convexe de l'espace ouvert}

\subsubsection{Enjeux et principe d'agrégation de points de mesures adjacents}

Les quatre indicateurs proposés précédemment sont calculés en tout point d'un échantillonnage ponctuel de l'espace ouvert. Afin de permettre une lecture synthétique des résultats, nous proposons une solution d'agrégation des points de mesure garantissant une information visuelle stable en chaque cellule. Cette proposition facilite en outre l'émergence de motifs géométriques caractérisables pour une meilleure compréhension des potentialités de l'espace urbain.

(Sarradin, 2004), d'après les travaux de (Peponis et al., 1997), rapporte que le champ visuel d'un observateur en mouvement dans l'espace ouvert urbain est exposé à deux types de changements. Ainsi, le changement continu ou changement de perspective des surfaces ne provoque-t-il aucune réelle modification de la topologie (aussi appelée aspect) de son univers visuel. A contrario, l'apparition ou la disparition d'éléments spatiaux (comme par exemple des extrémités de murs), aussi appelée changement discret ou transition, produit une modification substantielle de la topologie (un événement visuel) de son univers visuel, par introduction d'une discontinuité visible.

Cette distinction ayant été faite, (Peponis et al., 1997) a proposé de partitionner l'espace ouvert en fonction des événements visuels. Les méthodes qui en ont résulté ont conduit à décrire l'espace en configurations convexes ${ }^{4}$. Pour (Hillier et Hanson, 1984) rapporté par (Sarradin, 2004), les espaces convexes correspondent en effet à notre perception d'unités spatiales complètement disponibles à notre expérience. Dans ce contexte de découpage en sous-ensembles convexes, une transition est

\footnotetext{
${ }^{4}$ Une forme est dite convexe lorsque toute paire de points de celle-ci peut-être jointe par un segment de droite entièrement contenu dans la forme.
} 
assimilable au changement d'aspect de l'univers visuel d'un observateur en mouvement entre deux espaces convexes adjacents.

Les deux techniques de partitionnement déterministe (le s-partitionnement comme le e-partitionnement) de l'espace ouvert que nous présentons dans la suite de cet article, sont issues des travaux de (Peponis et al., 1997). Elles visent à produire un ensemble d'unités spatiales cohérentes au sens de la perception visuelle, construites à partir des événements visuels. L'hypothèse que nous formulons alors (et que nous vérifierons avec notre cas d'application nantais) consiste à considérer que ces deux techniques sont assimilables à des opérateurs d'agrégation compatibles au sens des quatre indicateurs de qualification du champ visuel présentés précédemment.

\subsection{2. s-segments et s-partitionnement}

La technique de s-partitionnement proposée par (Peponis et al., 1997) repose sur un principe général d'extension des arêtes concourantes en un sommet d'angle réflexe $e^{5}$. L'intention consiste à identifier un ensemble de s-lines (que nous appellerons dans la suite de cet article s-segments) et à en dériver un ensemble de sespaces ou éléments de s-partition, le «s » signifiant ici surface (ibid., p. 768). Ce principe, illustré en figure 6 , permet de construire un ensemble d'arêtes virtuelles par extension d'un sous-ensemble d'arêtes opaques, les s-segments, qui serviront ensuite (par le biais du mécanisme dit de polygonisation) à partitionner l'espace en un ensemble unique de polygones convexes (pour une configuration spatiale donnée) de manière parfaitement déterministe.

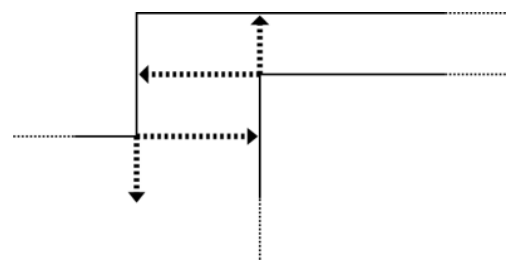

Figure 6. Principe d'extension des arêtes concourantes en chacun des angles réflexes. Ce mécanisme permet de créer les s-lines ou s-segments qui seront utilisés, lors de la phase de polygonisation, pour construire les éléments de s-partition.

Comme le constate (Peponis et al., 1997, p. 768) traduit par Sarradin (2004, p. 50), les éléments de s-partition issus de la polygonisation des s-segments sont construits de telle façon que, lorsque l'observateur passe d'un élément de s-partition à l'un de ses voisins immédiat, une nouvelle surface opaque apparaît dans ou disparait de son champ de vision. Ainsi, la transition d'un espace convexe à un autre

\footnotetext{
${ }^{5}$ Un angle réflexe est un angle dont la mesure est comprise entre $180^{\circ}$ et $360^{\circ}$.
} 
est marquée par le changement au sein des informations visuelles de l'environnement. La réciproque n'étant pas garantie (des surfaces ou parties de surfaces peuvent apparaître ou disparaître sans que l'observateur ait traversé une transition, (ibid.)), (Peponis et al., 1997) propose une technique de partitionnement alternative.

\subsection{3. e-segments et e-partitionnement}

Les s-espaces issus du s-partitionnement n'étant pas parfaitement stables (au sens d'une stabilité informationnelle du champ de vision), Peponis et al. (1997, p. 769) ont proposé une technique alternative dite de e-partitionnement, le «e » signifiant ici end-point. Celle-ci complète l'ensemble de s-segments issu de l'approche précédente (extension des angles réflexes) par un ensemble de linéaires issus de l'extension des diagonales. Une diagonale est ici entendue au sens d'un segment de droite reliant deux sommets d'arêtes opaques (discontinuités de type coin ou angle réflexe) sans traverser de murs (Sarradin, 2004, p. 51). Si ces diagonales peuvent être étendues, de part et d'autre, à l'intérieur de l'espace ouvert, à la manière de l'illustration en figure 7, ces extensions de discontinuités viennent alors compléter l'ensemble des ssegments déjà obtenu pour former le nouvel ensemble des e-lines (que nous appellerons dans la suite de cet article e-segments).

Comme le constate (Sarradin, 2004, p. 51), l'avantage de ce partitionnement est que non seulement la transition d'un espace convexe à un autre est marquée, comme pour le s-partitionnement, par un événement visuel, mais aussi que tous les événements visuels tels qu'ils ont été définis précédemment sont représentés par une transition entre des partitions.

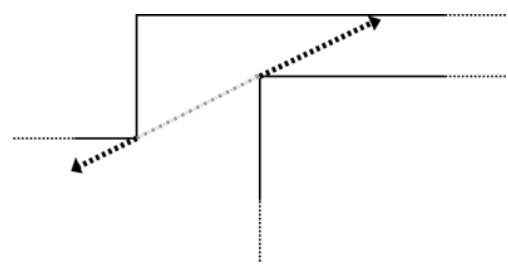

Figure 7. Principe d'extension des diagonales. Ce mécanisme, complétant celui qui consiste à étendre les angles réflexes, permet de créer les e-lines ou e-segments qui seront utilisés, lors de la phase de polygonisation, pour construire les éléments de epartition.

(Peponis et al., 1997, p. 770) affirme que l'ensemble des isovists générés à partir de points de vue d'un même e-espace recouvre les mêmes discontinuités et partage globalement les mêmes arêtes opaques. Par contre, tout changement de e-espace pour le point de vue, se traduit par une transformation radicale de l'isovist correspondant. Les auteurs ajoutent aussi que, du strict point de vue informationnel, il est probablement judicieux de regrouper les isovists dont les points de génération 
partagent un même e-espace (In other words, from the point of view of information about an environment, we may consider that: isovists can usefully be grouped according to sets which correspond to the points within e-spaces, (ibid.)). C'est en connaissance de cette assertion que nous avons formule l'hypothèse selon laquelle ces deux techniques de partitionnement de l'espace ouvert sont assimilables à des opérateurs d'agrégation compatibles au sens des quatre indicateurs de qualification du champ visuel présentés précédemment.

\section{Cas d'application}

\subsection{Contexte d'étude}

Dans le cadre de l'étude (Chauvat, 2012), déjà mentionnée, a été identifié un ensemble de terrains adaptés au «mouvement naturel » du piéton (dans le sens où les obstacles à cette mobilité sont limités), d'altimétrie et d'élévation à l'épannelage relativement constantes, ainsi que de composition architectonique homogène. Ces quatre terrains, tous situés dans le centre de Nantes, correspondent aux secteurs de la place Royale, des places du Change et de Sainte Croix, de la place du Bouffay et enfin de la place du Commerce. Ces places sont de configurations diverses (Manceau, 1981), ce qui permet d'éprouver les indicateurs avec différentes morphologies.

Pour le calcul du bassin de visibilité, étant donné le contexte urbain de cette étude, nous avons arrêté la portée du rayon de visibilité à $100 \mathrm{~m}$. Nous estimons en effet empiriquement que l'évaluation des différents indicateurs n'est pas significativement modifiée par la prise en compte d'une plus grande portée du champ de vision, au regard des rapports de distances observables dans cet environnement.

\subsection{Quantification du champ visuel en tous points}

Pour analyser les valeurs des quatre indicateurs nous avons procédé à un échantillonnage orthogonal inframétrique (la résolution planimétrique est de $50 \mathrm{~cm}$ par $50 \mathrm{~cm}$ ) des quatre secteurs. Les valeurs des points (de 135000 pour le secteur du Change et de Sainte Croix à 340000 pour celui de la place Royale) ont été « rasterisées » avant d'être visualisées à l'aide de QGIS. La planche de la figure 8 (seul le secteur de la place Royale est reproduit ici en raison de contraintes éditoriales) constitue une synthèse sous forme de cartes thématiques choroplèthes, par secteur, de ces traitements. Afin de faciliter la lecture des résultats, nous avons opté pour une classification par classes d'effectifs égaux. La symbologie procède du plus sombre (faibles valeurs) au plus clair (fortes valeurs). 

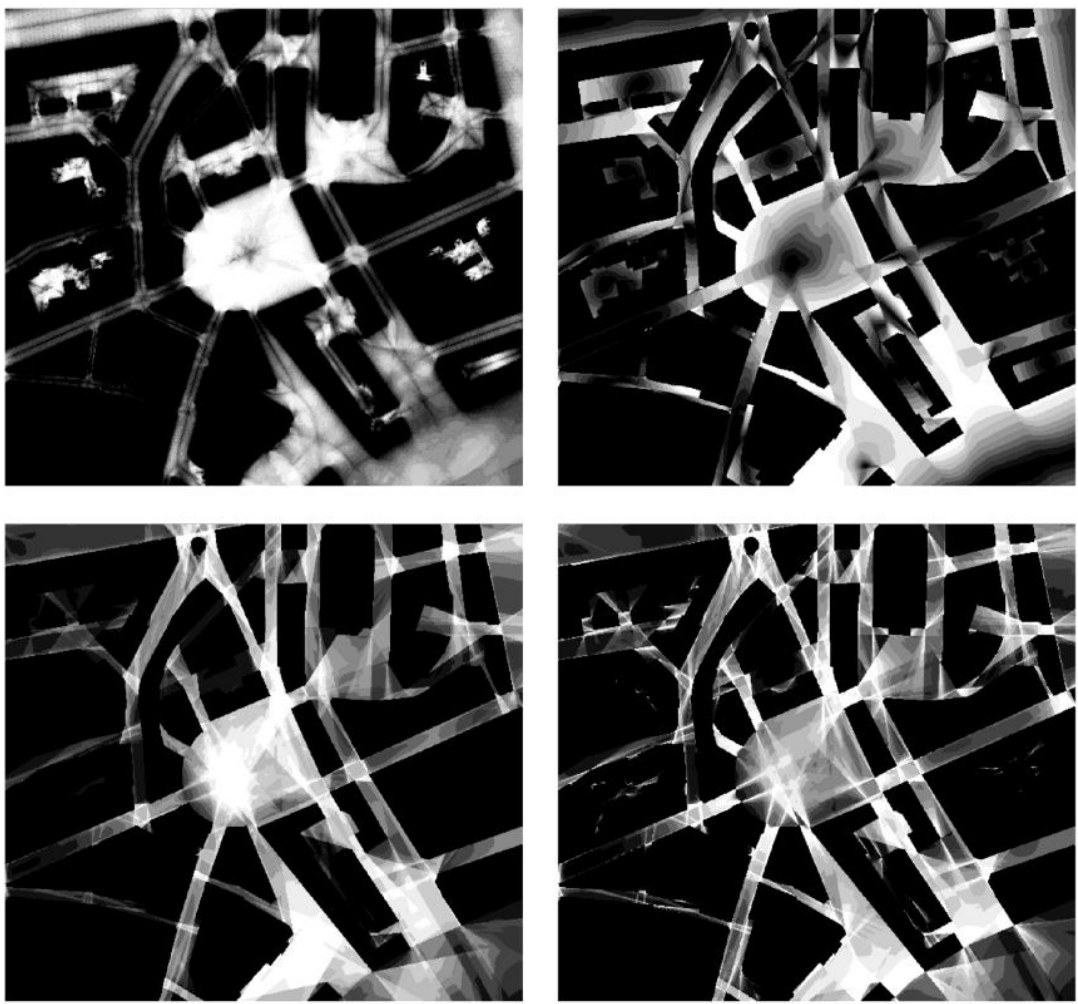

Figure 8. De haut en bas et de gauche à droite, pour le secteur de la place Royale, représentation de l'entropie, de l'excentricité, de l'occlusivité et de l'anticipation. La symbologie procède du plus sombre (faibles valeurs) au plus clair (fortes valeurs).

\subsection{Quantification du champ visuel à l'item de partition}

Considérons à présent les valeurs ponctuelles représentées dans le paragraphe précédent à travers le filtre d'un partitionnement convexe de l'espace visible. Cette agrégation produit respectivement la figure 9 (cas des s-partitions) et la figure 10 (cas des e-partitions). Pour une lecture plus intuitive, les cellules ne contenant aucun point, de taille négligeable, figurent en noir $100 \%$ et ont été retranchées lors des calculs statistiques. Elles génèrent toutefois, en termes de représentation cartographique, un certain bruit, perceptible notamment dans le cas de l'e-partition. La classification retenue est la même que celle qui a été utilisée pour la quantification en tout point. 

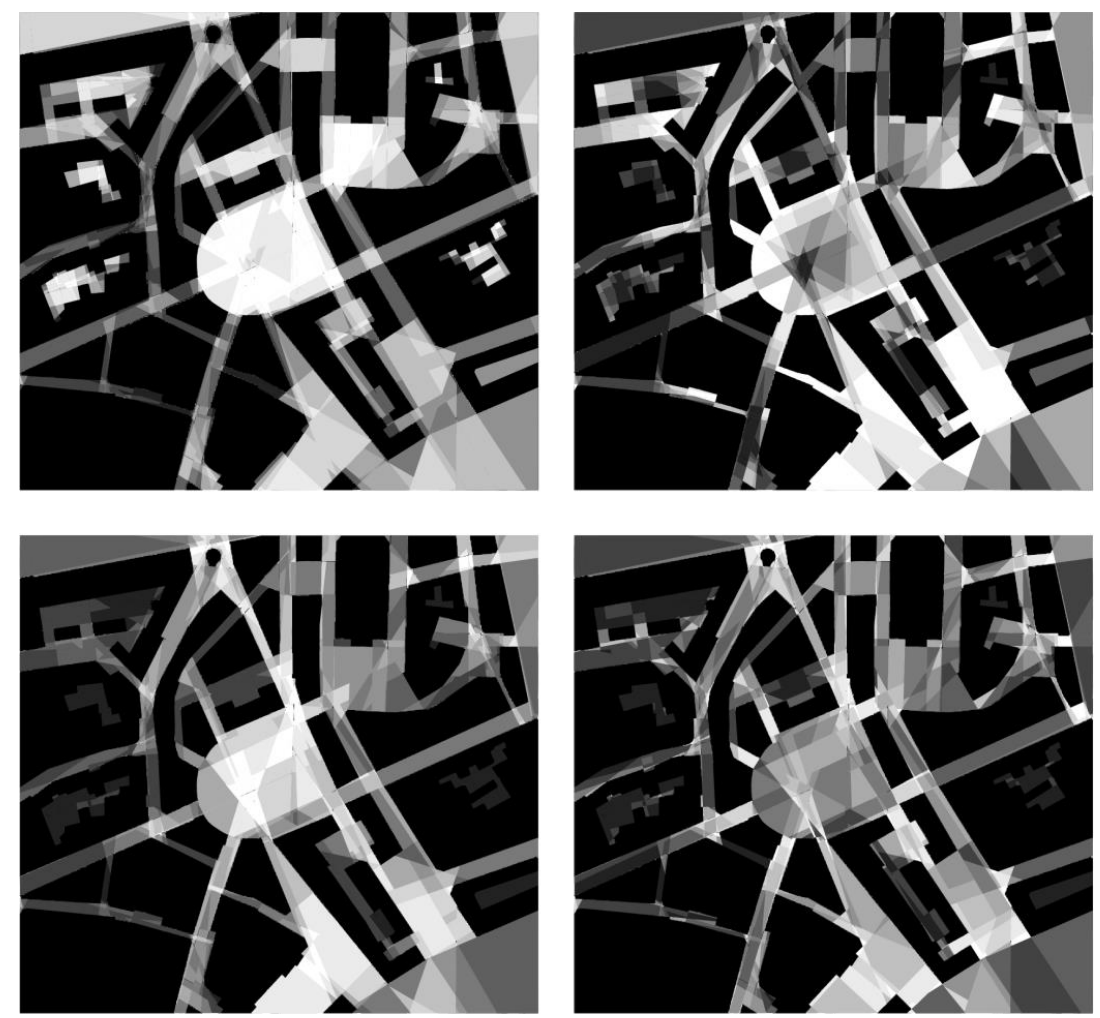

Figure 9. De haut en bas et de gauche à droite, pour le secteur de la place Royale, représentation de l'entropie, de l'excentricité, de l'occlusivité et de l'anticipation exprimés à travers le filtre d'un s-partitionnement. La symbologie procède du plus sombre (faibles valeurs) au plus clair (fortes valeurs).

Les données répertoriées dans le tableau 1 révèlent diverses informations relatives à la stabilité de l'agrégation opérée par les deux modes de partitionnement convexe. Elles seront analysées en 4.2. Le coefficient de variation moyen représente la valeur moyenne des rapports de l'écart-type sur la moyenne des valeurs pour chaque cellule du partitionnement. L'instabilité figure la sensibilité de chaque indicateur selon l'importance des surfaces des cellules. Cet indicateur est obtenu en rapportant la moyenne des coefficients de variation pondérés par la surface de la cellule au coefficient de variation moyen sans pondération. 

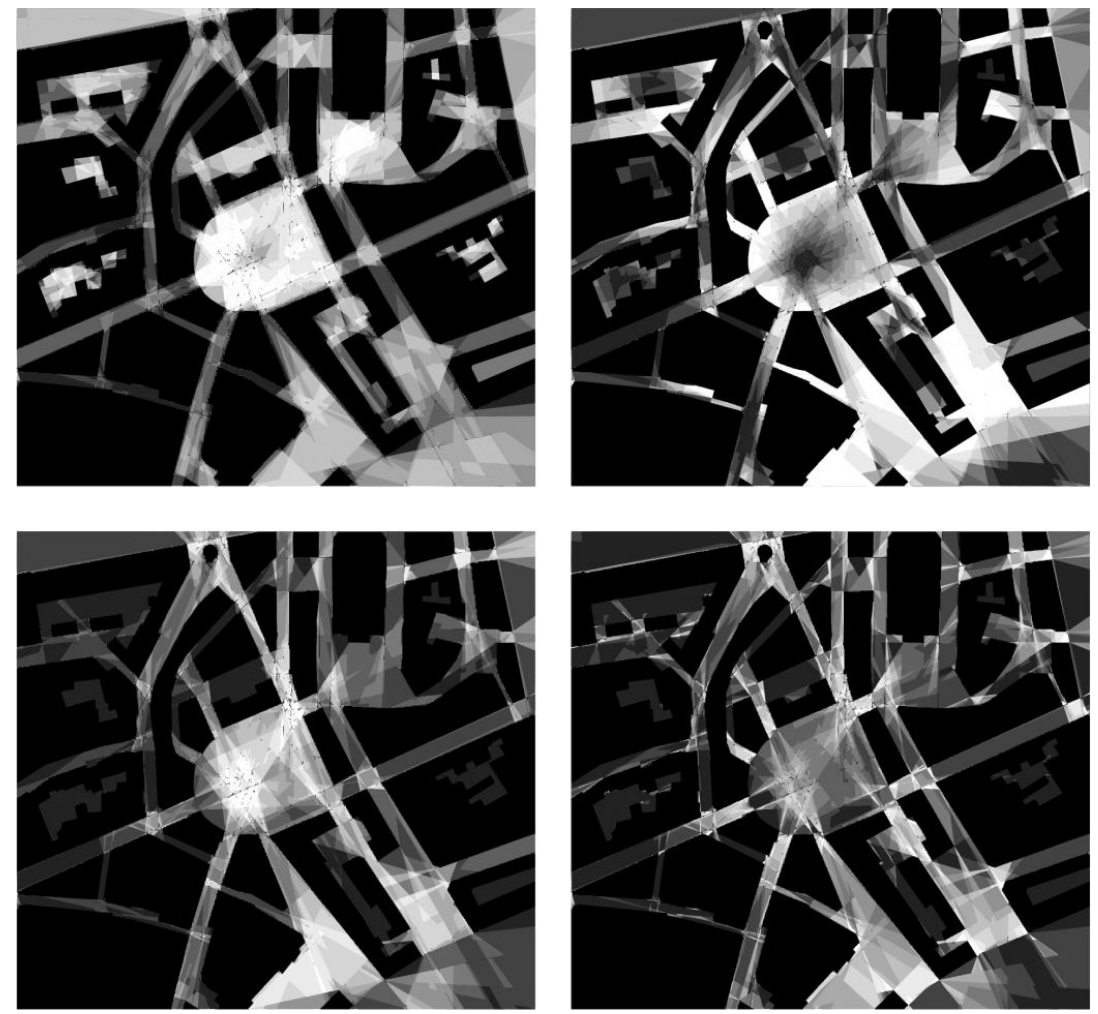

Figure 10. De haut en bas et de gauche à droite, pour le secteur de la place Royale, représentation de l'entropie, de l'excentricité, de l'occlusivité et de l'anticipation exprimés à travers le filtre d'un e-partitionnement. La symbologie procède du plus sombre (faibles valeurs) au plus clair (fortes valeurs).

Tableau 1. Indicateurs statistiques relatifs à la stabilité du procédé d'agrégation.

\begin{tabular}{|c|c|c|c|c|}
\cline { 2 - 5 } \multicolumn{1}{c|}{} & \multicolumn{2}{c|}{ S-partition } & \multicolumn{2}{c|}{ E-partition } \\
\cline { 2 - 5 } & $\begin{array}{c}\text { Coefficient de } \\
\text { variation } \\
\text { moyen }\end{array}$ & $\begin{array}{c}\text { Instabilité } \\
\text { (sensibilité au } \\
\text { paramètre } \\
\text { surface) }\end{array}$ & $\begin{array}{c}\text { Coefficient de } \\
\text { variation } \\
\text { moyen }\end{array}$ & $\begin{array}{c}\text { Instabilité } \\
\text { (sensibilité au } \\
\text { paramètre } \\
\text { surface) }\end{array}$ \\
\hline Entropie & 0,03 & 26,29 & 0,02 & 9,41 \\
\hline Excentricité & 0,13 & 39,31 & 0,06 & 14,15 \\
\hline Occlusivité & 0,09 & 34,10 & 0,02 & 13,49 \\
\hline Anticipation & 0,14 & 28,10 & 0,05 & 10,20 \\
\hline
\end{tabular}




\section{Discussion}

\subsection{Potentialités contrastées des indicateurs}

La qualification du bassin visuel observable depuis un point est susceptible d'influer sur le processus de conception d'un projet urbain ou architectural, de par l'évaluation de l'impact visuel d'un aménagement, la diversité des vues offertes, etc.

Les indicateurs présentés ici ont été choisis en raison d'une intuition initiale de cohérence entre la prévisibilité, le poids des masses visuelles et la part d'incertitude inhérente au contour immédiatement environnant d'une part, et le déplacement d'un piéton en situation de dérive (au sens de la théorie éponyme du situationniste Debord (1956)) d'autre part. Il s'agit d'une hypothèse forte mais également réductrice, un modèle de déplacement piéton en site urbain ne pouvant se suffire de la seule forme de l'environnement immédiat. Une première observation des résultats fait ressortir quelques saillances.

L'entropie, dessine une forme de squelette topologique de l'espace ouvert (illustrée en figures 8 et 11). Les points du squelette, plus foncés que ceux qui les environnent, sont ceux pour lesquels l'entropie est minimale (la prévisibilité du contour y est alors maximale). Ces points, sortes de promontoires au sens de la visibilité, sont, localement, ceux qui exposent le plus l'observateur qui s'y trouve mais ce sont aussi ceux qui lui permettent de mieux «anticiper» les éventuelles intrusions. Ce squelette disparaît avec un partitionnement convexe, la généralisation gommant cette singularité géométrique. L'agrégation met en valeur de vastes surfaces aux valeurs homogènes : l'entropie n'y varie que peu. Ces surfaces, de par leur importance, dépassent la logique du partitionnement convexe. Elles seraient caractéristiques de l'indicateur et permettraient de découper l'espace visible en un ensemble d'unités entropiques élémentaires.

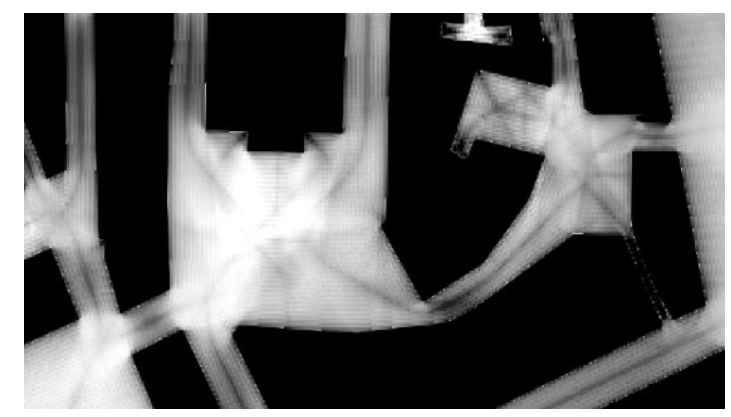

Figure 11. Zoom sur le quart nord-est du secteur de la place Royale (église Saint Nicolas). Représentation du champ scalaire de l'indicateur de prévisibilité du bassin de visibilité (entropie de Shannon de la fonction de distances radiales). L'entropie dessine une forme de squelette topologique de l'espace ouvert. La symbologie procède du plus sombre (faibles valeurs) au plus clair (fortes valeurs). 
Les zones matérialisées par l'excentricité sont moins précises. Elles révèlent des centralités ou, par le biais de lignes de fuite, des corridors de visibilité. Elles semblent aussi marquer des zones de stabilité de l'environnement visuel immédiat, zones qui seront affinées par les indicateurs d'occlusion (occlusivité et anticipation).

L'anticipation, dessine des aires de transitions marquées, représentées en figures 8 et 12. C'est-à-dire des lieux aux approches desquels la promesse d'un «bouleversement» de l'environnement visuel immédiat croît substantiellement. Ainsi, toute rue débouchant sur la place Royale comprend un point chaud à son extrémité (l'anticipation à proximité du point $\mathrm{A}$ en figure 12 est maximale). Le sujetpiéton est alors «poussé par la promesse » d'une découverte d'informations visuelles qui lui sont cachées. A contrario, les zones de minimum de potentiel d'anticipation, plus sombres (telle celle du point $B$ en figure 12), correspondent aux endroits où l'environnement immédiat est plus lisible et mieux maîtrisé en termes de perception des formes (la part de «surprise » possible y est moindre).

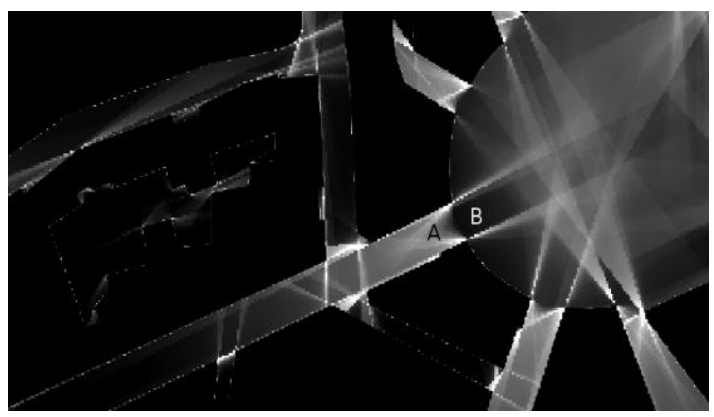

Figure 12. Zoom sur le quart sud-ouest du secteur de la place Royale (au débouché de la rue Crébillon sur la place Royale). Représentation du champ scalaire de l'indicateur d'anticipation. La symbologie procède du plus sombre (faibles valeurs) au plus clair (fortes valeurs).

Bien qu'étant toutes deux formalisées à partir des discontinuités visuelles, l'occlusivité et l'anticipation n'ont pas le même objectif. L'occlusivité évalue un potentiel global, soit la masse cachée d'un paysage urbain. On l'utilisera dès lors que l'on cherchera un point d'observation maximisant ou minimisant la part des occlusions (la signalétique en milieu urbain peut en être un exemple d'application). Pour un piéton, l'anticipation prendrait sens vis-à-vis d'une représentation cognitive de son environnement, induite par la morphologie du bâti alentours. Cet indicateur traiterait de la mobilité des piétons en milieu urbain. Il modéliserait une motivation intrinsèque à leur déplacement : la promesse d'une information cachée.4.2. Validité de l'hypothèse d'agrégation

De manière qualitative, on peut tout d'abord évoquer une forme de correspondance entre les figures 8 et 9 , ainsi qu'entre les figures 8 et 10. Le procédé 
de s-partitionnement gomme les singularités géométriques, visibles en figure 8 et résultant des définitions propres à chaque indicateur. Le e-partitionnement de la figure 10, par effet de maillage plus resserré, offre un rendu visuel très proche à la matrice infra-métrique de la figure 8. Les deux types d'agrégation généralisent l'information, la rendant ainsi plus aisément exploitable.

Afin de dépasser cette impression visuelle, nous avons calculé pour chaque indicateur un coefficient de variation moyen. Dans l'ensemble, les valeurs de ce coefficient sont relativement faibles. Il atteint au maximum $14 \%$ dans le cas de l'indicateur anticipation, pour un s-partitionnement. Le passage à un epartitionnement permet de diviser par deux l'instabilité de l'indicateur en chaque cellule. L'entropie est très stable, au regard des deux formes de partitionnement.

Pour une application plus robuste, nous avons observé l'évolution de cette instabilité en fonction de l'importance de la surface des cellules. Excentricité et occlusivité semblent réagir plus fortement à ce paramètre, alors que l'entropie et l'anticipation y sont moins sensibles. Ces deux derniers indicateurs seraient plus efficaces lors de traitements sur des partitions de grande taille.

Du fait de la bonne stabilité des quatre indicateurs, que ce soit après un spartitionnement ou un e-partitionnement, nous validons l'hypothèse selon laquelle ces méthodes permettraient une agrégation acceptable des valeurs des indicateurs calculées sur une trame initiale régulière de pas infra-métrique.

\subsection{Quelle application pour une conception raisonnée de l'environnement urbain ? Approche par la hiérarchisation des s-segments}

Les quatre indicateurs présentés dans cet article figurent autant d'outils pour une meilleure compréhension de l'espace urbain. L'occlusivité permet une étude de potentiel de visibilité propice à des applications de signalétique urbaine (par exemple), l'anticipation propose la description d'une motivation intrinsèque au déplacement d'un piéton, au même titre que l'excentricité et l'entropie, ainsi que l'a déjà relevé (Stamps, 2010). Le recours à un mécanisme de partitionnement de l'espace visible offre, quant à lui, une lecture plus cohérente de ces indicateurs. En effet, il permet une agrégation des valeurs et leur affectation à des cellules mesurables in situ. On donne alors une «épaisseur » à l'indicateur, celui-ci n'étant plus évalué en un point mais en une surface.

La s-partition présente des cellules aisément appréhendables dans un environnement urbain, plus grossières que celles issues de l'e-partition. La surface moyenne des s-espaces, plus importante que celle des e-espaces, semble mieux correspondre à l'échelle du sujet d'étude et des outils de l'analyste spatial.

Toutefois, la donnée vectorielle utilisée (les référentiels IGN) est très générique et sa résolution planimétrique est assez grossière pour l'usage que nous en faisons. L'échantillonnage au point (infra-métrique) et plus encore les techniques de partitionnement retenues sont d'une résolution supérieure à celle des référentiels. Il importe donc de rester prudent sur les implications spatiales précises de nos 
résultats. Nous pouvons d'ailleurs remarquer, à ce propos, qu'une étape préalable de simplification des empreintes de bâtiments aurait probablement supprimé quelques s-segments ou e-segments parasites, artéfacts liés aux techniques de partitionnement convexe utilisées.

Plus généralement, le choix d'un partitionnement convexe de l'espace visible, s'il permet une agrégation intéressante des indicateurs, doit toutefois être effectué selon les dimensions du territoire étudié. Les cellules résultantes doivent comporter une surface moyenne suffisante par rapport au niveau de définition de la donnée dont on dispose sur le site. De même, il s'agirait de négliger les cellules de forme trop étirée. A ce titre, le recours à un indicateur de compacité (caractérisant la circularité) permettrait une critique pertinente des résultats obtenus.

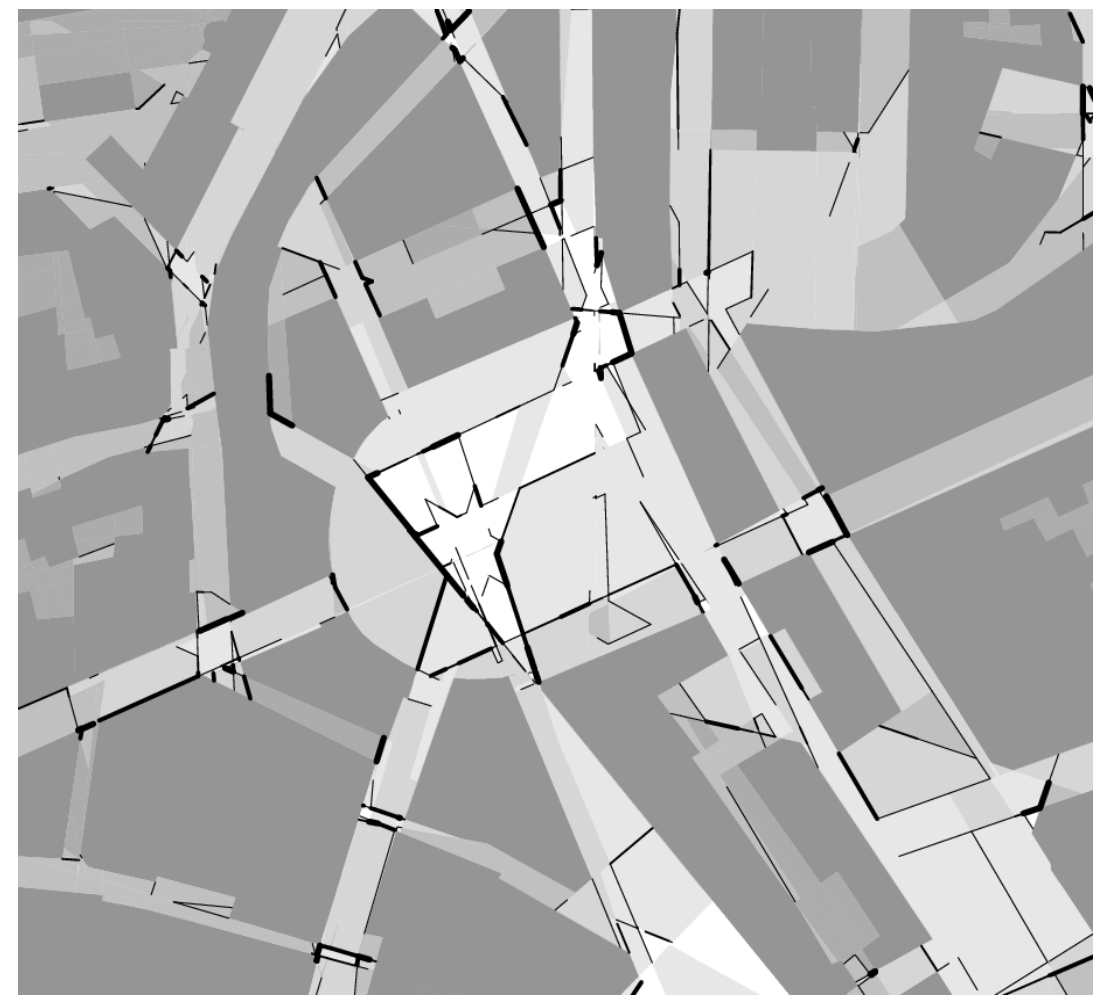

Figure 13. Pour le secteur de la place Royale, hiérarchisation des frontières discrètes (cas de l'occlusivité) issues d'un s-partitionnement.

Cette précaution étant prise, on peut distinguer des tendances globales pour les quatre indicateurs, repérables notamment par des gradients de couleur plus ou moins marqués. Si l'on s'interroge sur la structure « cachée » de l'espace, on peut associer 
chaque s-segment ou e-segment à une frontière discrète. En effet, chaque s-segment (ou e-segment) délimite des cellules d'information visuelle différente. On se propose de hiérarchiser les différents segments la composant. Pour ce faire, on classe chaque segment selon l'amplitude des valeurs qu'il sépare. Pour chaque indicateur, on peut ainsi tracer une carte de la géométrie structurant le territoire. A cet effet, les figures 13 et 14 illustrent cette hiérarchisation pour le cas de l'occlusivité (figure 13) et de l'anticipation (figure 14), pour un s-partitionnement. Bien que de formulations proches, ces deux indicateurs ne révèlent pas les mêmes structures. Les transitions discrètes ainsi lisibles, retranscriptions objectives de la géométrie du cadre bâti, peuvent être exploitées dans le cas d'une étude visant un aménagement cohérent de l'espace. On identifie de cette manière les seuils significatifs au cours d'une déambulation piétonne. Ces éléments pourraient être pris en compte pour la suggestion d'un parcours de découverte, suivant les orientations paysagères de ses concepteurs (révélation brutale ou douce d'objets d'intérêt, bassin de visibilité maximal ou contraint aidant à l'orientation, etc.). Ces exemples figurent à titre d'ouverture sur les potentialités offertes par les indicateurs en termes d'analyse de la part cachée d'un territoire urbain.

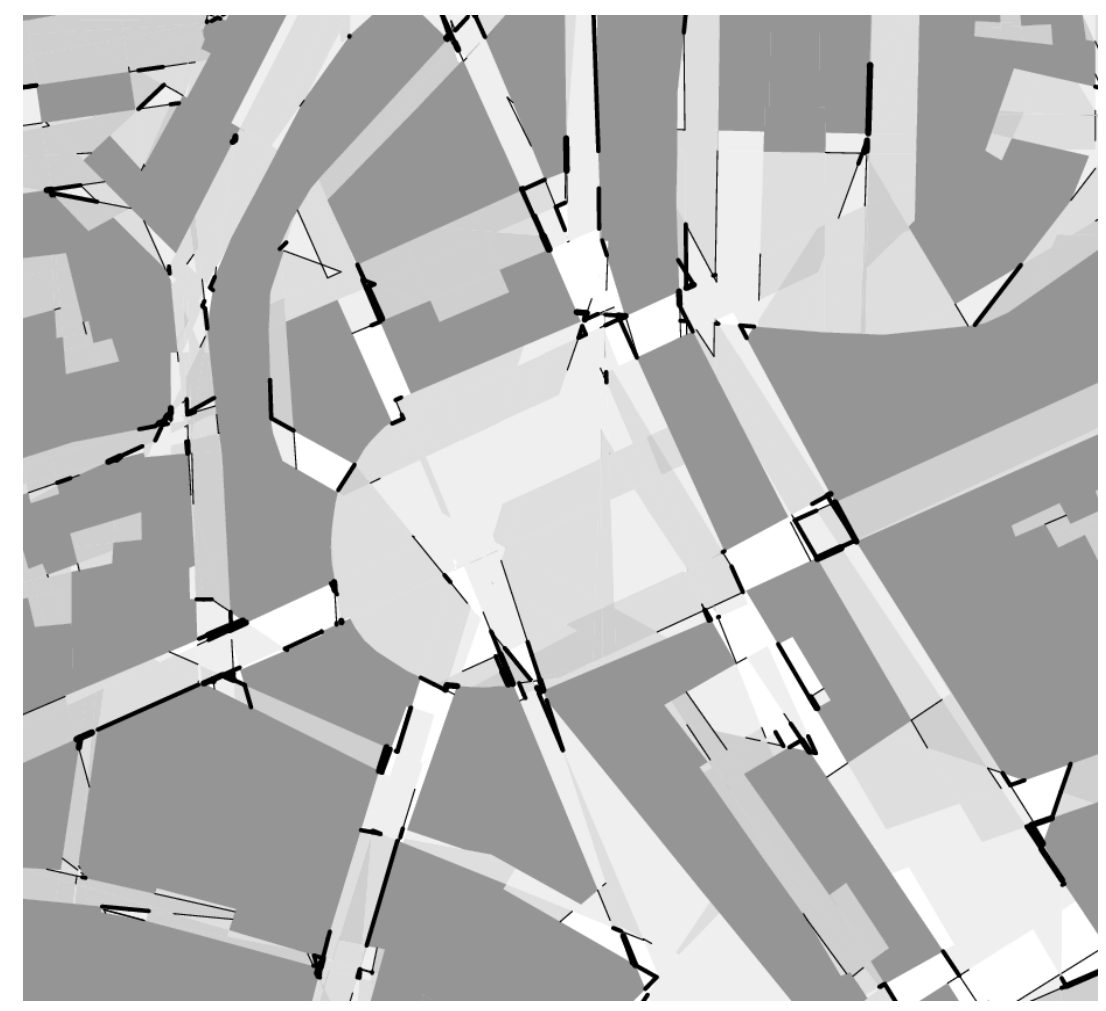

Figure 14. Pour le secteur de la place Royale, hiérarchisation des frontières discrètes (cas de l'anticipation) issues d'un s-partitionnement. 


\section{Conclusion}

L'étude présentée dans cet article s'est fixé pour but d'analyser de manière systématique et reproductible la part d'inconnu, d'incertitude, de l'environnement urbain immédiat d'un piéton. Elle a consisté en la mise en œuvre, dans le contexte d'un SIG, d'un modèle d'analyse (en tout point de l'espace ouvert) du champ visuel, analyse utilisant des jeux de données vectorielles standards. Ce modèle repose plus précisément sur deux principaux paramètres :

- l'évaluation d'un champ d'isovists partiels (la portée du champ de vision étant limitée) à l'aide de quatre indicateurs liés à la théorie de l'information (entropie de Shannon), au poids des masses visuelles (excentricité) ou à la part d'occlusions dans le bassin de visibilité (occlusivité et anticipation),

- le choix d'un principe de partitionnement convexe de l'espace visible, agrégeant les valeurs d'indicateurs en unités d'information visible stable.

Les résultats obtenus présentent diverses structurations de l'espace. La squelettisation, révélée par l'entropie, met en évidence des «promontoires » visuels. On pose également comme hypothèse l'existence d'unités entropiques élémentaires, structurant le milieu urbain. L'excentricité dessine des corridors visuels et des zones de stabilité au sens de la visibilité. L'anticipation enfin trace les contours de zones où la promesse d'un réel changement de l'environnement visuel peut orienter la mobilité d'un piéton en situation de dérive. Le recours à des techniques de partitionnement convexe (Peponis et al., 1997) permet une agrégation intéressante des résultats, donnant une épaisseur propice à une exploitation au service de l'aménagement d'un espace urbain.

Les combinaisons de ces indicateurs diffèrent selon les enjeux (sécurité visuelle, déplacement, lisibilité d'une séquence urbaine, surprise, etc.) et permettent une analyse plus approfondie de l'ambiance d'un espace urbain. La formulation d'un indicateur global de «qualité visuelle » reste cependant difficilement envisageable en pratique, du fait de ces enjeux contradictoires et du caractère subjectif de certains paramètres.

Cette analyse comportementale contextualisée d'un fragment urbain pourrait utilement être validée par un dispositif expérimental in situ (processus d'enquête et préférences déclarées ou préférences révélées par les trajectoires (Foltête et Piombini, 2010 ; Piombini et al., 2014)) en prenant garde à la complexité et à la multiplicité des motivations intrinsèques au déplacement.

\section{Bibliographie}

Adolphe L. (2001). A simplified model of urban morphology: application to an analysis of the environmental performance of cities. Environment and Planning B: Planning and Design, 28(2), 183-200. doi:10.1068/b2631

Batty M., Jiang B. (1999). Multi-agent simulation: new approaches to exploring space-time dynamics in GIS. London (UK). 
Benedikt M. L. (1979). To take hold of space: isovists and isovist fields. Environment and Planning B: Planning and Design, 6(1), 47-65.

Brayer L. (2013). Filmer l'ambiance urbaine: Les dispositifs vidéographiques à l'œuvre chez William H. Whyte dans La vie sociale des petits espaces urbains. Ambiances [En ligne], Représentation - Traduction - Ecriture. http://ambiances.revues.org/335

Cauvin, C. (1999). Pour une approche de la cognition spatiale intra-urbaine. Cybergeo: European Journal of Geography [En ligne], Politique, Culture, Représentations, document 72, mis en ligne le 27 janvier 1999. URL : http://cybergeo.revues.org/5043 ; DOI : 10.4000/cybergeo.5043 doi:10.4000/cybergeo.5043

Chauvat G. (2012). Cartographier le mystère: caractérisation d'un indicateur figurant la propension au mouvement d'un individu en situation de dérive (p. 72). Nantes, France.

Conroy Dalton R., Dalton N. (2001). OmniVista: an application for Isovist field and path analysis. 3rd International Space Syntax Symposium. Atlanta, Georgia, USA.

Cullen G. (1961). Townscape (p. 315). New York: Reinhold Pub. Corp.

Debord G.-E. (1956). Théorie de la dérive. Internationale Situationniste, 2, 19-23.

Edussuriya P., Chan A., Ye A. (2011). Urban morphology and air quality in dense residential environments in Hong Kong. Part I: District-level analysis. Atmospheric Environment, 45(27), 4789-4803. doi:10.1016/j.atmosenv.2009.07.061

Foltête J.-C., Piombini A. (2010). Deviations in pedestrian itineraries in urban areas: a method to assess the role of environmental factors. Environment and Planning B: Planning and Design, 37(4), 723-739. doi:10.1068/b35015

Hall E. T. (1966). The hidden dimension. Doubleday. Retrieved from http://www.bookforfree.us/PDF/The-Hidden-Dimension-BY-Edward-T.-Hall-ID3764.pdf

Hillier, B., Hanson, J. (1984). The Social Logic of Space. Cambridge: Cambridge University Press. doi:10.1017/CBO9780511597237

Hillier B., Penn A., Hanson J., Grajewski T., Xu J. (1993). Natural movement: or, configuration and attraction in urban pedestrian movement. Environment and Planning B: Planning and Design, 20(1), 29-66. doi:10.1068/b200029

Joliveau T. (2004). Géomatique et gestion environnementale du territoire. Recherches sur un usage géographique des SIG. Mémoire d'Habilitation à Diriger des Recherches en Sciences Humaines. Université de Rouen, France.

Kaplan R. (1973). Predictors of environmental preference: designers and clients. In W. F. E. Preiser (Ed.), EDRA 4: Fourth International EDRA Conference - The Environmental Design Research Association (Vol. 1, pp. 265-274).

Leduc T., Kontovourkis O. (2012). Towards a mixed approach combining visibility and mobility studies to analyze the eleftheria square, Nicosia (CY). In R. Billen, M. Caglioni, O. Marina, G. Rabino, R. S. José (Eds.), 3D Issues in Urban and Environmental Systems (pp. 67-77). Bologna, Italy: Societa' Editrice Esculapio.

Llobera M. (2003). Extending GIS-based visual analysis: the concept of visualscapes. International Journal of Geographical Information Science, 17(1), 25-48.

Manceau, D. (1981). Echantillon d'espaces urbains de l'agglomération nantaise (p. 114). Nantes, France. 
Morello E., Ratti C. (2009). A digital image of the city: 3D isovists in Lynch's urban analysis. Environment and Planning B: Planning and Design, 36(5), 837-853.

Péneau J.-P. (2000). Les ambiances urbaines. In M.-F. Mattei, D. Pumain (Eds.), Données Urbaines (pp. 375-386). Paris, France: Anthropos.

Peponis J., Wineman J., Rashid M., Hong Kim S., Bafna S. (1997). On the description of shape and spatial configuration inside buildings: convex partitions and their local properties. Environment and Planning B: Planning and Design, 24(5), 761-781.

Piombini, A., Leduc, T., Woloszyn, P. (2014). Usage de la morphométrie dans la révélation des préférences de mobilité. Application aux cheminements piétons. Revue Internationale de Géomatique, 24(1), 101-130. doi:10.3166/rig.24.101-130

Rana S. (2006). Isovist Analyst - An Arcview extension for planning visual surveillance. ESRI International User Conference. (on CD-ROM) (Vol. 1, p. 9). Redlands, USA.

Salat S. (2011). Les villes et les formes: Sur l'urbanisme durable (p. 544). Hermann.

Sarradin F. (2004). Analyse morphologique des espaces ouverts urbains le long de parcours. Thèse de l'Université de Nantes, ED MTGC, laboratoire CERMA, école nationale supérieure d'architecture de Nantes, France.

Stamps III, A. E. (2005). Isovists, enclosure, and permeability theory. Environment and Planning B: Planning and Design, 32(5), 735-762.

Stamps III, A. E. (2010). Slines, entropy, and environmental exploration. Environment and Planning B: Planning and Design, 37(4), 704-722. doi:10.1068/b35117

Suleiman W., Joliveau T., Favier E. (2012). Une nouvelle méthode de calcul d'isovist en 2 et 3 dimensions. In R. Billen, M. Binard, P. Hallot, J.-P. Donnay (Eds.), Actes de la Conférence internationale de Géomatique et Analyse Spatiale - SAGEO 2012 (pp. 366386). doi:2268/133126

Turner A., Doxa M., O’Sullivan D., Penn A. (2001). From isovists to visibility graphs: a methodology for the analysis of architectural space. Environment and Planning B: Planning and Design, 28(1), 103-121. 